УДК 548.1:537.226.4

\title{
Phases of Anionic Ordering in Elpasolite Structures (Ordered Perovskites)
}

\author{
Roman G. Sevryukov* \\ Ivan N.Safonov ${ }^{\dagger}$ \\ Siberian Federal University \\ Svobodny, 79, Krasnoyarsk, 660041 \\ Russia \\ Maksim S. Molokeev \\ Kirensky Institute of Physics SB RAS \\ Akademgorodok, 50/38, Krasnoyarsk, 660036 \\ Far Eastern State Transport University \\ Serysheva, 47, Khabarovsk, 680021 \\ Russia \\ Sergey V. Misyul ${ }^{\S}$ \\ Siberian Federal University \\ Svobodny, 79, Krasnoyarsk, 660041 \\ Russia
}

Received 23.11.2015, received in revised form 09.12.2015, accepted 20.01.2016

The 108 dissymmetric phases were obtained as a result of $X$ anions ordering in elpasolite structure $A_{2} B B^{\prime} X_{6}$ (ordered perovskite) using group theoretical methods. The critical order parameters which transform according to the irreducible representations of $\Gamma$ and $\mathrm{X}$ points of Brillouin zone of $\mathrm{O}_{h}^{5}-F m \overline{3} m$ space group were considered only. Polyhedral structures showed for dissymmetric phases which are accompanied by appearing of ferroelectricity at phase transition. All results are summarized in convenient tables.

Keywords: elpasolite, structural data, a complete condensate of order parameters, irreducible representations, critical distortion, phase transition, dissymmetric phases.

DOI: 10.17516/1997-1397-2016-9-1-108-118.

\section{Introduction}

A lot of compounds crystallized in space group $O_{h}^{5}-F m \overline{3} m$, part of them have important practical properties. Of particular interest are the numerous perovskite-like compounds with space group $F m \overline{3} m$ which undergo many phase transitions (PT) during changing of external influence $[1,2]$. The crystals of elpasolite with general formula $A_{2} B B^{\prime} X_{6}\left(A, B, B^{\prime}\right.$ - cations, $X$ - anion) are intensively studied at present and belong to this group of compounds. Such

\footnotetext{
*srg06.6@mail.ru

$\dagger$ isafonov@sfu-kras.ru

†msmolokeev@mail.ru

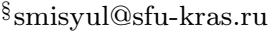

(c) Siberian Federal University. All rights reserved
} 
compounds can be found among halides, oxides, oxohalides, cyanides, hydrides. The cations $A$ and $\mathrm{B}$ can be $\mathrm{NH}_{4}$ ammonium group also.

In elpasolites also called ordered perovskites there are two kinds of ionic groups $B X_{6}$ and $B^{\prime} X_{6}$, which alternate along the three 4-fold axes (Fig. 1a), contrary to simple perovskite with equivalent octahedra only. Thus, elpasolite cubic cell can be considered as perovskite cell with doubled cell parameters. Cryolite $A_{3} B^{\prime} X_{6}$ is a special case of elpasolite structure, where $A$ and $B$ ions in $A_{2} B B^{\prime} X_{6}$ are the same.

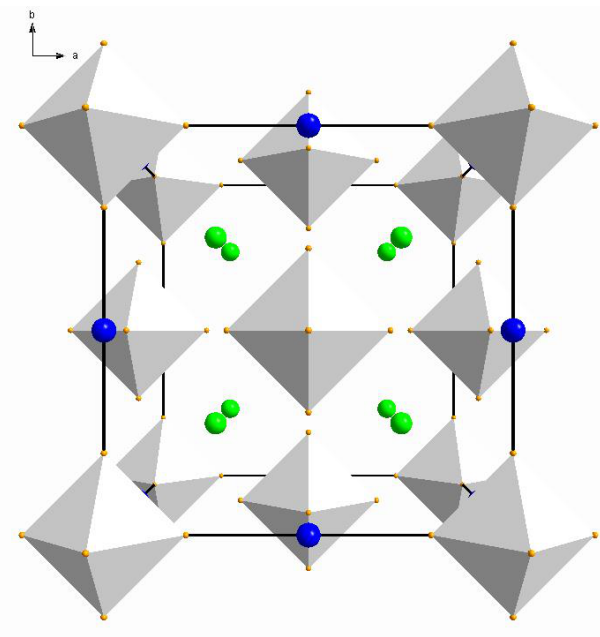

(a) $X$ anion located in $24 e$ position

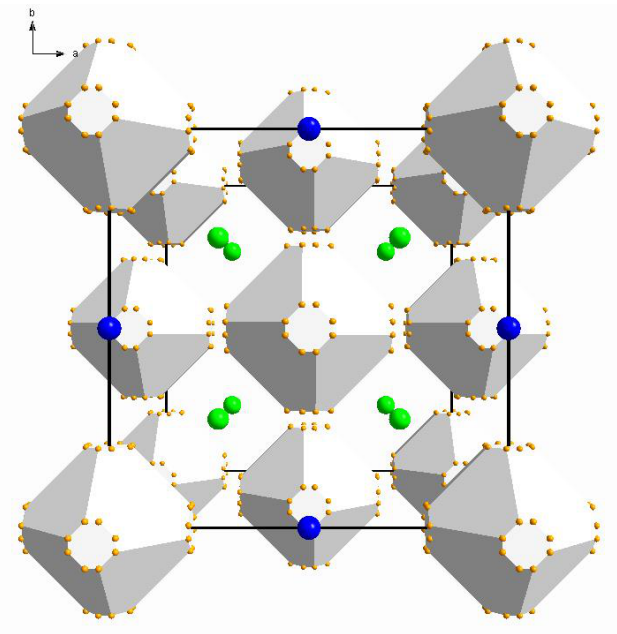

(b) $X$ is disordered, $192 l$ site

Fig. 1. $A_{2} B B^{\prime} X_{6}$ structure. Octahedra $B^{\prime} X_{6}$ has gray color (ion $B^{\prime}$ is located in the center of octahedron), ions $B$ - marked by blue color, ions $A$ - marked by green color

Most PT, which observed in these compounds, are described as rotational distortions associated with small rotations of octahedra groups $B X_{6}$ and $B^{\prime} X_{6}[1,2]$. As a rule such PT are displacive type. Relatively recent research of ammonium elpasolites $\left(\mathrm{NH}_{4}\right)_{2} \mathrm{KGaF}_{6}$ [3] and $\left(\mathrm{NH}_{4}\right)_{2} \mathrm{KWO}_{3} \mathrm{~F}_{3}$ [4], solid solutions of ammonium cryolites $\left(\mathrm{NH}_{4}\right)_{3} \mathrm{Ga}_{1-x} \mathrm{Sc}_{x} \mathrm{~F}_{6}$ [5-7], oxyfluorides $\left(\mathrm{NH}_{4}\right)_{3} \mathrm{WO}_{3} \mathrm{~F}_{3},\left(\mathrm{NH}_{4}\right)_{3} \mathrm{TiOF}_{5}[8,9]$ showed that at $\mathrm{PT}$ the ordering of atoms and groups including octahedral groups occur, i.e. PT is order-disorder type.

In the study of phase transitions in elpasolite compounds a well established scheme is used, on the first stage of which the group-theoretical analysis of possible distortions of structure is carried out [10]. Such analysis allows one to obtain all possible space groups of distorted phrases, correctly choose model of distorted structure of low-symmetry phase, describe the behavior of physical properties.

A lot of works are devoted to symmetric analysis of the crystals with the space group $F m \overline{3} m$ [10-14]. Without going into details of the results of these works, it should be noted that space groups of distorted (dissymmetric) phases induced by all irreducible representations (IR) of Lifshitz points of Brillouin zone are obtained. The mechanical representation assuming rigid, linked and undistorted at $\mathrm{PT}$ octahedral ions $B X_{6}$ and $B^{\prime} X_{6}[10,11]$ was investigated to analyze experimental works about $\mathrm{PT}$ in halide elpasolites and cryolites. It should be mentioned the paper [13], which deals with the permutation and mechanical representations with the same space group. Let us recall that the basic functions of mechanical representation are the displacements of atoms of structure, but permutation representation - scalar values, which 
in the case of PT order-disorder type are associated with the relative probabilities of atoms to occupy a certain position in the crystal.

Thus, the permutation representation of corresponding structure should be analyzed for consideration of the order-disorder PT. The analysis of representation means: 1) determination of the composition, i.e. which IR and how many times they are included in permutation representation and 2) definition of basic functions of IR, which are included in permutation representation. Such work was done in [13]. In this paper we will use the results of [13].

Experimental [3-9] and theoretical investigations [9-11] of $A_{2} B B^{\prime} X_{6}$ crystals prove that order parameters (OP) at PT, which are associated to ordering of $X$ anions, are transformed by IR of center, point $\Gamma$, and boundary, point X, of Brillouin zone (Tab. 1) of space group $F m \overline{3} m$ (designation of IR are in accordance with reference books $[15,16])$. IR and OP, which induce symmetry changes, are commonly known as critical or primary.

Table 1. Lifshitz points of Brillouin zone of space group $F m \overline{3} m$. The main periods of primitive lattice: $\mathbf{a}_{\mathbf{1}}=\tau(0,1,1), \mathbf{a}_{\mathbf{2}}=\tau(1,0,1), \mathbf{a}_{\mathbf{3}}=\tau(1,1,0)$, edge of face-centered cube $a=2 \tau$. The main periods of reciprocal lattice: $\mathbf{b}_{\mathbf{1}}=\pi / \tau(-1,1,1), \mathbf{b}_{\mathbf{2}}=\pi / \tau(1,-1,1)$, $\mathbf{b}_{\mathbf{3}}=\pi / \tau(1,1,-1)$

\begin{tabular}{cc}
\hline Type of star from $[15,16]$ & Rays of star \\
\hline $\mathbf{k}_{\mathbf{1}}=\frac{1}{2}\left(\mathbf{b}_{\mathbf{1}}+\mathbf{b}_{\mathbf{2}}\right)=\frac{\pi}{\tau}(0,0,1)$, \\
$\mathbf{k}_{\mathbf{2}}=\frac{1}{2}\left(\mathbf{b}_{\mathbf{1}}+\mathbf{b}_{\mathbf{3}}\right)=\frac{\pi}{\tau}(0,1,0)$, \\
$\mathbf{k}_{\mathbf{3}}=\frac{1}{2}\left(\mathbf{b}_{\mathbf{2}}+\mathbf{b}_{\mathbf{3}}\right)=\frac{\pi}{\tau}(1,0,0)$ \\
\hline $\mathbf{k}_{\mathbf{1}}=0$ \\
\hline
\end{tabular}

However, the distortion of the structure of the parent phase in some cases is impossible to describe using only critical OP. In the distorted (dissymmetric) phase the appeared displacements or ordering of ions can be consistent with the symmetry of the phase and they are controlled by non-critical (secondary) OP and IR. All OP, critical and non-critical, which are appeared at PT, form a complete condensate of OP [17]. Symmetry analysis indicates the presence and type of non-critical OP only. Numerical values of both critical and non-critical distortions and OP, which participate in complete condensate, are determined from experimental, in the best case structural, data.

The purpose of the current work is to obtain and visualize structures of dissymmetric phases which appeared in result of $X$ anion ordering in elpasolite crystals $A_{2} B B^{\prime} X_{6}$. For the reasons stated above, the dissymmetric structures were considered which are associated with critical OP transformed by IR of $\Gamma$ and X points of Brillouin zone (Tab. 1) of space group $F m \overline{3} m$. Herewith non-critical OP and IR are not considered. The work about non-critical deformations will be presented in further publications.

The results of [13] and program ISOTROPY [18] were used to achieve this goal. Crystal structures of dissymmetric phases were presented using program Diamond [19]. 


\section{Parent disordered phase $\mathrm{A}_{2} \mathrm{BB}^{\prime} \mathrm{X}_{6}$}

The results of works [7-9], allow us to suppose that in the parent phase $G_{0}$ of elpasolite $A_{2} B B^{\prime} X_{6}$ there are 24 anions $X$, which can be disordered among $192 l$ site of cubic face-centered cell of space group $F m \overline{3} m$ [20]. The probability for $X$ anion to occupy some site is equal to 0.125 . Polyhedral representation of such disordered structure is showed in Fig. 1b, coordinates of ions of $A_{2} B B^{\prime} X_{6}$ are in Tab. 2.

According to the work [13], one can argue that all IR of Lifshitz points of Brilloiun zone of $F m \overline{3} m$ group are included in permutation representation on $192 l$ site at which the $X$ anion is disordered. The IR matrixes for $\Gamma$ and X points of Brillouin zone of $F m \overline{3} m$ space group are in Tab. 3. The IR matrixes are showed for generators of group only in accordance with reference books [15]: $h_{2}$ - rotation on $180^{\circ}$ around axis $[1,0,0] ; h_{3}$ - rotation on $180^{\circ}$ around axis $[0,1,0]$; $h_{5}-$ rotation on $240^{\circ}$ around axis $[1,1,1] ; h_{9}-$ rotation on $120^{\circ}$ around axis $[1,1,1] ; h_{13}-$ rotation on $180^{\circ}$ around axis $[-1,1,0] ; h_{25}$ - inversion.

Table 2. Coordinates of atoms of cubic elpasolite phase $A_{2} B B^{\prime} X_{6}$ (sp.gr. $F m \overline{3} m$ ). For $X$ anion there is coordinates in $192 l$ disordered general position (upper string) and average value in $24 e$ position (lower string)

\begin{tabular}{cccccc}
\hline Atom & Site & $x / a$ & $y / b$ & $z / c$ & Occupancy \\
\hline$B^{\prime}$ & $4 a$ & 0 & 0 & 0 & 1 \\
\hline$B$ & $4 b$ & 0.5 & 0.5 & 0.5 & 1 \\
\hline$A$ & $8 c$ & 0.25 & 0.25 & 0.25 & 1 \\
\hline \multirow{2}{*}{$X$} & $192 l$ & 0.015 & 0.05 & 0.20 & 0.125 \\
\cline { 2 - 5 } & $24 e$ & 0 & 0 & 0.20 & 1 \\
\hline
\end{tabular}

Notes. The type of IR is defined by two numbers divided by dash: first value is number of star of $k_{i}$ vector, second value defines sequence number of IR of this vector. In addition to notation of IR, corresponding to [15], there are notations which is accepted in foreign literature from [16]. The IR matrixes are showed for generators of group only in accordance with reference books [15]. The diagonal matrixes are presented in form of column with elements corresponding to elements on the main diagonal of matrixes. The sign «t» defines two IR, the matrix presents in first IR with sign «+», and in second IR with sign «-». The letters were used for the matrixes in form:

$$
I=\left(\begin{array}{cc}
1 & 0 \\
0 & 1
\end{array}\right), Q=\left(\begin{array}{cc}
1 & 0 \\
0 & -1
\end{array}\right), W=\left(\begin{array}{cc}
-1 / 2 & \sqrt{3} / 2 \\
-\sqrt{3} / 2 & -1 / 2
\end{array}\right), A=\left(\begin{array}{lll}
0 & 0 & 1 \\
1 & 0 & 0 \\
0 & 1 & 0
\end{array}\right), B=\left(\begin{array}{ccc}
1 & 0 & 0 \\
0 & 0 & 1 \\
0 & 1 & 0
\end{array}\right)
$$

\section{Ordered phases in elpasolites $\mathrm{A}_{2} \mathrm{BB}^{\prime} \mathrm{X}_{6}$}

The following Tab. 4 and Fig. 2 shows the results of a symmetry analysis, which includes space groups of ordered phases of $A_{2} B B^{\prime} X_{6}$ structure. As a critical OP were chosen only those, which are transformed by IR related to the $\Gamma$ and $\mathrm{X}$ stars of Brillouin zone of face-centered cubic cell of $F m \overline{3} m$ group. 
Table 3. Complete IR of $F m \overline{3} m$ group for Lifshitz points $\Gamma$ and $\mathrm{X}$ of Brillouin zone

\begin{tabular}{|c|c|c|c|c|c|c|c|c|}
\hline IR & $a_{1}$ & $a_{2}$ & $a_{3}$ & $\left(h_{2} / 0\right)$ & $\left(h_{3} / 0\right)$ & $\left(h_{5} / 0\right)$ & $\left(h_{13} / 0\right)$ & $\left(h_{25} / 0\right)$ \\
\hline $\begin{array}{l}10-1, X_{1}^{+} \\
10-2, X_{1}^{-}\end{array}$ & $\left(\begin{array}{c}-1 \\
-1 \\
1\end{array}\right)$ & $\left(\begin{array}{c}-1 \\
1 \\
-1\end{array}\right)$ & $\begin{array}{c}1 \\
-1 \\
-1\end{array}$ & $\left(\begin{array}{l}1 \\
1 \\
1\end{array}\right)$ & $\left(\begin{array}{l}1 \\
1 \\
1\end{array}\right)$ & $A$ & $B$ & $\pm\left(\begin{array}{l}1 \\
1 \\
1\end{array}\right)$ \\
\hline $\begin{array}{l}10-3, X_{3}^{+} \\
10-4, X_{3}^{-}\end{array}$ & $\left(\begin{array}{c}-1 \\
-1 \\
1\end{array}\right)$ & $\left(\begin{array}{c}-1 \\
1 \\
-1\end{array}\right)$ & $\left.\begin{array}{c}1 \\
-1 \\
-1\end{array}\right)$ & $\left(\begin{array}{c}-1 \\
-1 \\
1\end{array}\right)$ & $\left(\begin{array}{c}-1 \\
1 \\
-1\end{array}\right)$ & $A$ & $-B$ & $\pm\left(\begin{array}{l}1 \\
1 \\
1\end{array}\right)$ \\
\hline $\begin{array}{l}10-5, X_{2}^{+} \\
10-6, X_{2}^{-}\end{array}$ & $\left(\begin{array}{c}-1 \\
-1 \\
1\end{array}\right)$ & $\left(\begin{array}{c}-1 \\
1 \\
-1\end{array}\right)$ & $\begin{array}{c}1 \\
-1 \\
-1\end{array}$ & $\left(\begin{array}{l}1 \\
1 \\
1\end{array}\right)$ & $\left(\begin{array}{l}1 \\
1 \\
1\end{array}\right)$ & $A$ & $-B$ & $\pm\left(\begin{array}{l}1 \\
1\end{array}\right)$ \\
\hline $\begin{array}{l}10-7, X_{4}^{+} \\
10-8, X_{4}^{-}\end{array}$ & $\left(\begin{array}{c}-1 \\
-1 \\
1\end{array}\right)$ & $\left(\begin{array}{c}-1 \\
1 \\
-1\end{array}\right)$ & $\begin{array}{c}1 \\
-1 \\
-1\end{array}$ & $\left(\begin{array}{c}-1 \\
-1 \\
1\end{array}\right)$ & $\left(\begin{array}{c}-1 \\
1 \\
-1\end{array}\right)$ & $A$ & $B$ & $\pm\left(\begin{array}{l}1 \\
1 \\
1\end{array}\right)$ \\
\hline $\begin{array}{c}10-9, X_{5}^{+} \\
10-10, X_{5}^{-}\end{array}$ & $\left(\begin{array}{c}-1 \\
-1 \\
1\end{array}\right)$ & $\left(\begin{array}{c}-1 \\
-1 \\
1\end{array}\right)$ & $\begin{array}{c}-1 \\
-1 \\
1\end{array}$ & $\left(\begin{array}{c}-1 \\
-1 \\
1\end{array}\right)$ & $\left(\begin{array}{c}-1 \\
-1 \\
1\end{array}\right)$ & $\left(\begin{array}{c}-1 \\
-1 \\
1\end{array}\right)$ & $\left(\begin{array}{c}-1 \\
-1 \\
1\end{array}\right)$ & $\left(\begin{array}{c}-1 \\
-1 \\
1\end{array}\right)$ \\
\hline $\begin{array}{l}11-1, \Gamma_{1}^{+} \\
11-2, \Gamma_{1}^{-}\end{array}$ & 1 & 1 & 1 & 1 & 1 & 1 & 1 & \pm 1 \\
\hline $\begin{array}{l}11-3, \Gamma_{2}^{+} \\
11-4, \Gamma_{2}^{-}\end{array}$ & 1 & 1 & 1 & 1 & 1 & 1 & -1 & \pm 1 \\
\hline $\begin{array}{l}11-5, \Gamma_{3}^{+} \\
11-6, \Gamma_{3}^{-}\end{array}$ & $I$ & $I$ & $I$ & $I$ & $I$ & $W$ & $Q$ & $\pm I$ \\
\hline $\begin{array}{l}11-7, \Gamma_{5}^{+} \\
11-8, \Gamma_{5}^{-}\end{array}$ & $\left(\begin{array}{l}1 \\
1 \\
1\end{array}\right)$ & $\left(\begin{array}{l}1 \\
1 \\
1\end{array}\right)$ & $\left(\begin{array}{l}1 \\
1 \\
1\end{array}\right)$ & $\left(\begin{array}{c}-1 \\
-1 \\
1\end{array}\right)$ & $\left(\begin{array}{c}-1 \\
1 \\
-1\end{array}\right)$ & $A$ & $B$ & $\pm\left(\begin{array}{l}1 \\
1 \\
1\end{array}\right)$ \\
\hline $\begin{array}{c}11-9, \Gamma_{4}^{+} \\
11-10, \Gamma_{4}^{-}\end{array}$ & $\left(\begin{array}{l}1 \\
1 \\
1\end{array}\right)$ & $\left(\begin{array}{l}1 \\
1 \\
1\end{array}\right)$ & $\left(\begin{array}{l}1 \\
1 \\
1\end{array}\right)$ & $\left(\begin{array}{c}-1 \\
-1 \\
1\end{array}\right)$ & $\left(\begin{array}{c}-1 \\
1 \\
-1\end{array}\right)$ & $A$ & $-B$ & $\pm\left(\begin{array}{l}1 \\
1 \\
1\end{array}\right)$ \\
\hline
\end{tabular}

Table 4. Probable dissymmetric phases with $X$ ions ordering in elpasolite $A_{2} B B^{\prime} X_{6}$ structure. The cell parameters of dissymmetric phases are presented in terms of translation vectors of cubic face-centered unit cell of parent phase: $\mathbf{a}_{\mathbf{1}}^{\prime}=2 \tau(1,0,0), \mathbf{a}_{\mathbf{2}}^{\prime}=2 \tau(0,1,0), \mathbf{a}_{\mathbf{3}}^{\prime}=2 \tau(0,0,1)$

\begin{tabular}{lclll}
\hline \multirow{2}{*}{ Critical IR } & \multirow{2}{*}{$\begin{array}{c}\text { Critical values } \\
\text { of order parameters }\end{array}$} & \multicolumn{2}{c}{ Subgroup } & Basis \\
\cline { 3 - 4 } & & Schoenflies & International & \\
\hline $11-2, \Gamma_{1}^{-}$ & $(a)$ & $O^{3}$ & $F 432$ & $(1,0,0),(0,1,0),(0,0,1)$ \\
$11-3, \Gamma_{2}^{+}$ & $(a)$ & $T_{h}^{3}$ & $F m \overline{3}$ & $(1,0,0),(0,1,0),(0,0,1)$ \\
$11-4, \Gamma_{2}^{-}$ & $(a)$ & $T_{d}^{2}$ & $F \overline{4} 3 m$ & $(1,0,0),(0,1,0),(0,0,1)$ \\
$11-5, \Gamma_{3}^{+}$ & $(a, 0)$ & $D_{4 h}^{17}$ & $I 4 / m m m$ & $\left(\frac{1}{2}, \frac{1}{2}, 0\right),\left(\frac{\overline{1}}{2}, \frac{1}{2}, 0\right),(0,0,1)$ \\
\hline
\end{tabular}




\begin{tabular}{|c|c|c|c|c|}
\hline \multirow{2}{*}{ Critical IR } & \multirow{2}{*}{$\begin{array}{l}\text { Critical values } \\
\text { of order parameters }\end{array}$} & \multicolumn{2}{|c|}{ Subgroup } & \multirow{2}{*}{ Basis } \\
\hline & & Schoenflies & International & \\
\hline & $(a, b)$ & $D_{2 h}^{23}$ & Fmmm & $(1,0,0),(0,1,0),(0,0,1)$ \\
\hline \multirow[t]{3}{*}{$11-6, \Gamma_{3}^{-}$} & $(a, 0)$ & $D_{2 d}^{9}$ & $I \overline{4} m 2$ & $\left(\frac{1}{2}, \frac{1}{2}, 0\right),\left(\frac{\overline{1}}{2}, \frac{1}{2}, 0\right),(0,0,1)$ \\
\hline & $(0, a)$ & $D_{4}^{9}$ & $I 422$ & $\left(\frac{1}{2}, \frac{1}{2}, 0\right),\left(\frac{1}{2}, \frac{1}{2}, 0\right),(0,0,1)$ \\
\hline & $(a, b)$ & $D_{2}^{7}$ & $F 222$ & $(1,0,0),(0,1,0),(0,0,1)$ \\
\hline \multirow[t]{4}{*}{$11-7, \Gamma_{5}^{+}$} & $(a, 0,0)$ & $D_{2 h}^{25}$ & Immm & $\left(\frac{1}{2}, \frac{1}{2}, 0\right),\left(\frac{1}{2}, \frac{1}{2}, 0\right),(0,0,1)$ \\
\hline & $(a, a, a)$ & $D_{3 d}^{5}$ & $R \overline{3} m$ & $\left(\frac{\overline{1}}{2}, \frac{1}{2}, 0\right),\left(0, \frac{\overline{1}}{2}, \frac{1}{2}\right),(1,1,1)$ \\
\hline & $(a, a, b)$ & $C_{2 h}^{3}$ & $C 2 / m$ & $\left(\frac{\overline{1}}{2}, 1, \frac{\overline{1}}{2}\right),\left(\frac{\overline{1}}{2}, 0, \frac{1}{2}\right),\left(\frac{1}{2}, 0, \frac{1}{2}\right)$ \\
\hline & $(a, b, c)$ & $C_{i}^{1}$ & $P \overline{1}$ & $\left(0, \frac{1}{2}, \frac{1}{2}\right),\left(\frac{1}{2}, 0, \frac{1}{2}\right),\left(\frac{1}{2}, \frac{1}{2}, 0\right)$ \\
\hline \multirow[t]{6}{*}{$11-8, \Gamma_{5}^{-}$} & $(a, 0,0)$ & $D_{2 d}^{11}$ & $I \overline{4} 2 m$ & $\left(\frac{1}{2}, \frac{1}{2}, 0\right),\left(\frac{1}{2}, \frac{1}{2}, 0\right),(0,0,1)$ \\
\hline & $(a, a, 0)$ & $C_{2 v}^{20}$ & $\operatorname{Imm} 2$ & $\left(\frac{1}{2}, 0, \frac{1}{2}\right),(0,1,0),\left(\frac{1}{2}, 0, \frac{1}{2}\right)$ \\
\hline & $(a, a, a)$ & $D_{3}^{7}$ & $R 32$ & $\left(\frac{\overline{1}}{2}, \frac{1}{2}, 0\right),\left(0, \frac{1}{2}, \frac{1}{2}\right),(1,1,1)$ \\
\hline & $(a, b, 0)$ & $C_{s}^{3}$ & $\mathrm{Cm}$ & $(0,0, \overline{1}),(0,1,0),\left(\frac{1}{2}, 0, \frac{1}{2}\right)$ \\
\hline & $(a, a, b)$ & $C_{2}^{3}$ & $C 2$ & $\left(\frac{1}{2}, 1, \frac{\overline{1}}{2}\right),\left(\frac{1}{2}, 0, \frac{1}{2}\right),\left(\frac{1}{2}, 0, \frac{1}{2}\right)$ \\
\hline & $(a, b, c)$ & $C_{1}^{1}$ & $P 1$ & $\left(0, \frac{1}{2}, \frac{1}{2}\right),\left(\frac{1}{2}, 0, \frac{1}{2}\right),\left(\frac{1}{2}, \frac{1}{2}, 0\right)$ \\
\hline \multirow[t]{4}{*}{$11-9, \Gamma_{4}^{+}$} & $(a, 0,0)$ & $C_{4 h}^{5}$ & $I 4 / m$ & $\left(0, \frac{1}{2}, \frac{1}{2}\right),\left(0, \frac{1}{2}, \frac{1}{2}\right),(1,0,0)$ \\
\hline & $(a, a, 0)$ & $C_{2 h}^{3}$ & $C 2 / m$ & $\left(\frac{\overline{1}}{2}, \frac{1}{2}, 1\right),\left(\frac{\overline{1}}{2}, \frac{\overline{1}}{2}, 0\right)\left(\frac{1}{2}, \overline{\frac{1}{2}}, 0\right)$ \\
\hline & $(a, a, a)$ & $C_{3 i}^{2}$ & $R \overline{3}$ & $\left(\frac{\overline{1}}{2}, \frac{1}{2}, 0\right),\left(0, \frac{\overline{1}}{2}, \frac{1}{2}\right),(1,1,1)$ \\
\hline & $(a, b, c)$ & $C_{i}^{1}$ & $P \overline{1}$ & $\left(0, \frac{1}{2}, \frac{1}{2}\right),\left(\frac{1}{2}, 0, \frac{1}{2}\right),\left(\frac{1}{2}, \frac{1}{2}, 0\right)$ \\
\hline \multirow[t]{6}{*}{$11-10, \Gamma_{4}^{-}$} & $(a, 0,0)$ & $C_{4 v}^{9}$ & $I 4 \mathrm{~mm}$ & $\left(0, \frac{1}{2}, \frac{1}{2}\right),\left(0, \frac{1}{2}, \frac{1}{2}\right),(1,0,0)$ \\
\hline & $(a, a, 0)$ & $C_{2 v}^{20}$ & $\operatorname{Imm} 2$ & $\left(\frac{1}{2}, \frac{1}{2}, 0\right),(0,0,1),\left(\frac{1}{2}, \frac{1}{2}, 0\right)$ \\
\hline & $(a, a, a)$ & $C_{3 v}^{5}$ & $R 3 m$ & $\left(\frac{\overline{1}}{2}, \frac{1}{2}, 0\right),\left(0, \frac{\overline{1}}{2}, \frac{1}{2}\right),(1,1,1)$ \\
\hline & $(a, b, 0)$ & $C_{s}^{3}$ & $\mathrm{Cm}$ & $(\overline{1}, 0,0),(0,0,1),\left(\frac{1}{2}, \frac{1}{2}, 0\right)$ \\
\hline & $(a, a, b)$ & $C_{s}^{3}$ & $\mathrm{Cm}$ & $\left(\frac{1}{2}, \frac{1}{2}, 1\right),\left(\frac{1}{2}, \frac{1}{2}, 0\right),\left(\frac{1}{2}, \frac{1}{2}, 0\right)$ \\
\hline & $(a, b, c)$ & $C_{1}^{1}$ & $P 1$ & $\left(0, \frac{1}{2}, \frac{1}{2}\right),\left(\frac{1}{2}, 0, \frac{1}{2}\right),\left(\frac{1}{2}, \frac{1}{2}, 0\right)$ \\
\hline \multirow[t]{4}{*}{$10-1, \mathrm{X}_{1}^{+}$} & $(a, 0,0)$ & $D_{4 h}^{1}$ & $P 4 / m m m$ & $\left(\frac{1}{2}, 0, \frac{1}{2}\right),\left(\frac{1}{2}, 0, \frac{1}{2}\right),(0,1,0)$ \\
\hline & $(a, a, a)$ & $O_{h}^{1}$ & $P m \overline{3} m$ & $(1,0,0),(0,1,0),(0,0,1)$ \\
\hline & $(a, b, a)$ & $D_{4 h}^{1}$ & $P 4 / m m m$ & $(0,1,0),(0,0,1),(1,0,0)$ \\
\hline & $(a, b, c)$ & $D_{2 h}^{1}$ & Pmmm & $(1,0,0),(0,1,0),(0,0,1)$ \\
\hline \multirow[t]{2}{*}{$10-2, \mathrm{X}_{1}^{-}$} & $(a, 0,0)$ & $D_{4 h}^{4}$ & $P 4 / n n c$ & $\left(\frac{1}{2}, 0, \frac{1}{2}\right),\left(\frac{1}{2}, 0, \frac{1}{2}\right),(0,1,0)$ \\
\hline & $(a, 0, a)$ & $D_{4 h}^{3}$ & $P 4 / n b m$ & $(0,1,0),(0,0,1),(1,0,0)$ \\
\hline
\end{tabular}




\begin{tabular}{|c|c|c|c|c|}
\hline \multirow{2}{*}{ Critical IR } & \multirow{2}{*}{$\begin{array}{l}\text { Critical values } \\
\text { of order parameters }\end{array}$} & \multicolumn{2}{|c|}{ Subgroup } & \multirow{2}{*}{ Basis } \\
\hline & & Schoenflies & International & \\
\hline & $(a,-a, a)$ & $O^{1}$ & $P 432$ & $(1,0,0),(0,1,0),(0,0,1)$ \\
\hline & $(a, 0, b)$ & $D_{2 h}^{4}$ & Pban & $(0,1,0),(0,0,1),(1,0,0)$ \\
\hline & $(a, b, a)$ & $D_{4}^{1}$ & $P 422$ & $(0,1,0),(0,0,1),(1,0,0)$ \\
\hline & $(a, b, c)$ & $D_{2}^{1}$ & $P 222$ & $(1,0,0),(0,1,0),(0,0,1)$ \\
\hline \multirow[t]{4}{*}{$10-3, \mathrm{X}_{3}^{+}$} & $(a, 0,0)$ & $D_{4 h}^{6}$ & $P 4 / m n c$ & $\left(\frac{1}{2}, 0, \frac{1}{2}\right),\left(\frac{1}{2}, 0, \frac{1}{2}\right),(0,1,0)$ \\
\hline & $(a, 0, a)$ & $D_{4 h}^{12}$ & $P 4_{2} / n n m$ & $(0,1,0),(0,0,1),(1,0,0)$ \\
\hline & $(a,-a, a)$ & $T_{h}^{2}$ & $\operatorname{Pn} \overline{3}$ & $(1,0,0),(0,1,0),(0,0,1)$ \\
\hline & $(a, b, c)$ & $D_{2 h}^{2}$ & Pnnn & $(1,0,0),(0,1,0),(0,0,1)$ \\
\hline \multirow[t]{6}{*}{$10-4, \mathrm{X}_{3}^{-}$} & $(a, 0,0)$ & $D_{4 h}^{7}$ & $P 4 / n m m$ & $\left(\frac{\overline{1}}{2}, 0, \frac{1}{2}\right),\left(\frac{1}{2}, 0, \frac{1}{2}\right),(0,1,0)$ \\
\hline & $(a, 0, a)$ & $D_{4 h}^{10}$ & $\mathrm{P} 4_{2} / \mathrm{mcm}$ & $(0,1,0),(0,0,1),(1,0,0)$ \\
\hline & $(a, a, a)$ & $T_{d}^{1}$ & $P \overline{4} 3 m$ & $(1,0,0),(0,1,0),(0,0,1)$ \\
\hline & $(a, 0, b)$ & $D_{2 h}^{3}$ & Pccm & $(0,1,0),(0,0,1),(1,0,0)$ \\
\hline & $(a, b, a)$ & $D_{2 d}^{1}$ & $P \overline{4} 2 m$ & $(0,1,0),(0,0,1),(1,0,0)$ \\
\hline & $(a, b, c)$ & $D_{2}^{1}$ & $P 222$ & $(1,0,0),(0,1,0),(0,0,1)$ \\
\hline \multirow[t]{4}{*}{$10-5, \mathrm{X}_{2}^{+}$} & $(a, 0,0)$ & $D_{4 h}^{14}$ & $P 4_{2} / m n m$ & $\left(\frac{\overline{1}}{2}, 0, \frac{1}{2}\right),\left(\frac{1}{2}, 0, \frac{1}{2}\right),(0,1,0)$ \\
\hline & $(a, 0, a)$ & $D_{4 h}^{1}$ & $P 4 / m m m$ & $(0,1,0),(0,0,1),(1,0,0)$ \\
\hline & $(a,-a, a)$ & $T_{h}^{1}$ & $\operatorname{Pm} \overline{3}$ & $(1,0,0),(0,1,0),(0,0,1)$ \\
\hline & $(a, b, c)$ & $D_{2 h}^{1}$ & Pmmm & $(1,0,0),(0,1,0),(0,0,1)$ \\
\hline \multirow[t]{6}{*}{$10-6, \mathrm{X}_{2}^{-}$} & $(a, 0,0)$ & $D_{4 h}^{15}$ & $P 4_{2} / n m c$ & $\left(\frac{1}{2}, 0, \frac{1}{2}\right),\left(\frac{1}{2}, 0, \frac{1}{2}\right),(0,1,0)$ \\
\hline & $(a, 0, a)$ & $D_{4 h}^{3}$ & $P 4 / n b m$ & $(0,1,0),(0,0,1),(1,0,0)$ \\
\hline & $(a, a, a)$ & $T_{d}^{1}$ & $P \overline{4} 3 m$ & $(1,0,0),(0,1,0),(0,0,1)$ \\
\hline & $(a, 0, b)$ & $D_{2 h}^{4}$ & Pban & $(0,1,0),(0,0,1),(1,0,0)$ \\
\hline & $(a, b, a)$ & $D_{2 d}^{1}$ & $P \overline{4} 2 m$ & $(0,1,0),(0,0,1),(1,0,0)$ \\
\hline & $(a, b, c)$ & $D_{2}^{1}$ & $P 222$ & $(1,0,0),(0,1,0),(0,0,1)$ \\
\hline \multirow[t]{4}{*}{$10-7, X_{4}^{+}$} & $(a, 0,0)$ & $D_{4 h}^{9}$ & $P 4_{2} / m m c$ & $\left(\frac{1}{2}, 0, \frac{1}{2}\right),\left(\frac{1}{2}, 0, \frac{1}{2}\right),(0,1,0)$ \\
\hline & $(a, a, a)$ & $O_{4 h}^{4}$ & $P n \overline{3} m$ & $(1,0,0),(0,1,0),(0,0,1)$ \\
\hline & $(a, b, a)$ & $D_{4 h}^{12}$ & $P 4_{2} / n n m$ & $(0,1,0),(0,0,1),(1,0,0)$ \\
\hline & $(a, b, c)$ & $D_{2 h}^{2}$ & Pnnn & $(1,0,0),(0,1,0),(0,0,1)$ \\
\hline \multirow[t]{3}{*}{$10-8, \mathrm{X}_{4}^{-}$} & $(a, 0,0)$ & $D_{4 h}^{12}$ & $P 4_{2} / n n m$ & $\left(\frac{\overline{1}}{2}, 0, \frac{1}{2}\right),\left(\frac{1}{2}, 0, \frac{1}{2}\right),(0,1,0)$ \\
\hline & $(a, 0, a)$ & $D_{4 h}^{10}$ & $\mathrm{P}_{2} / \mathrm{mcm}$ & $(0,1,0),(0,0,1),(1,0,0)$ \\
\hline & $(a,-a, a)$ & $O^{2}$ & $P 4_{2} 32$ & $(1,0,0),(0,1,0),(0,0,1)$ \\
\hline
\end{tabular}




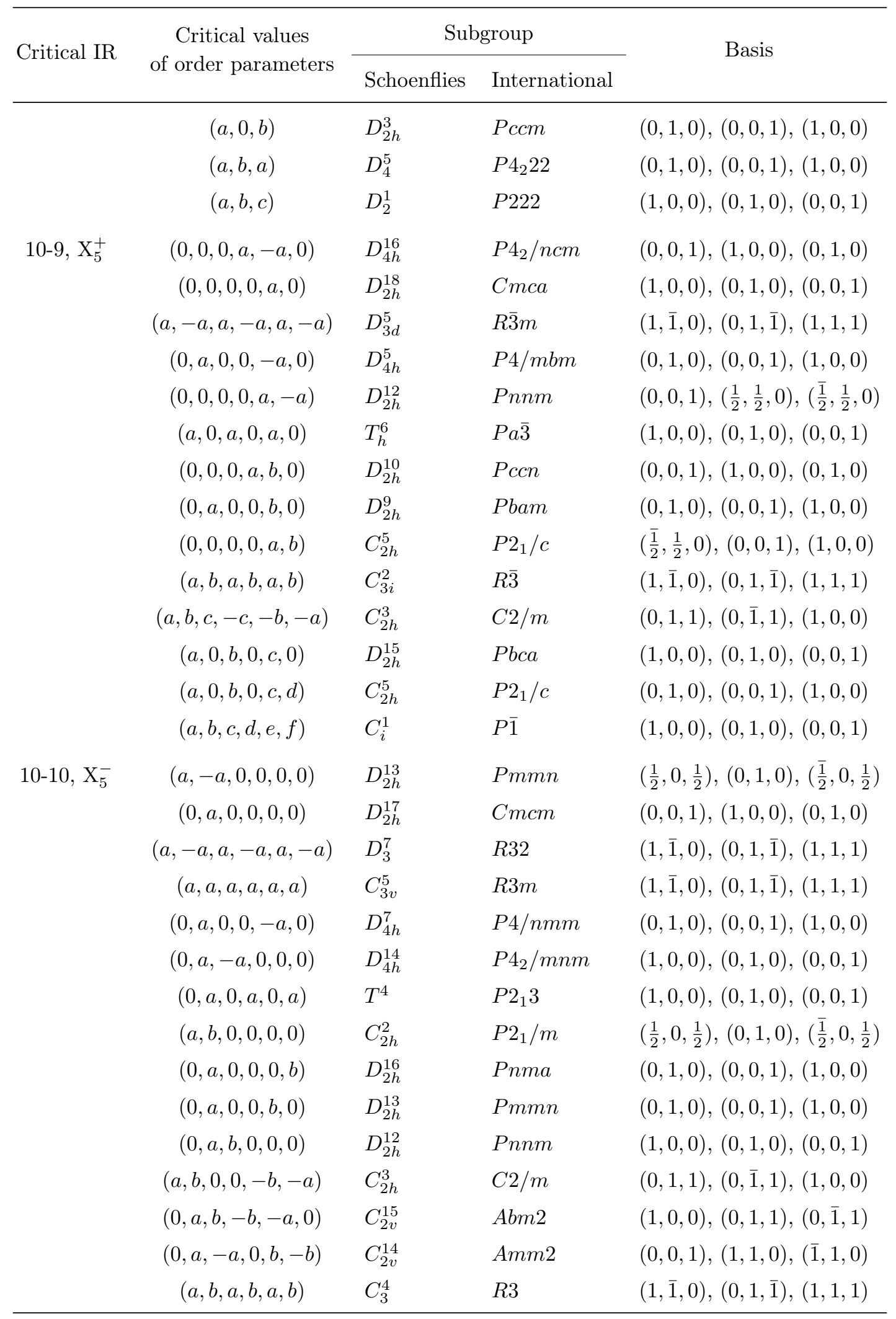




\begin{tabular}{ccccc}
\hline \multirow{2}{*}{ Critical IR $\begin{array}{c}\text { Critical values } \\
\text { of order parameters }\end{array}$} & \multicolumn{2}{c}{ Subgroup } & Basis \\
\cline { 2 - 3 } & Schoenflies & International & \\
\hline$(0, a, 0,0, b, c)$ & $C_{2 h}^{2}$ & $P 2_{1} / m$ & $(0,1,0),(0,0,1),(1,0,0)$ \\
$(0, a, b, c, 0,0)$ & $C_{2 h}^{5}$ & $P 2_{1} / c$ & $(0, \overline{1}, 0),(1,0,0),(0,1,1)$ \\
$(a, b, c,-c,-b,-a)$ & $C_{2}^{3}$ & $C 2$ & $(0,1,1),(0, \overline{1}, 1),(1,0,0)$ \\
$(a, b, c, c,-b,-a)$ & $C_{s}^{3}$ & $C m$ & $(0,1,1),(0, \overline{1}, 1),(1,0,0)$ \\
$(0, a, 0, b, 0, c)$ & $D_{2}^{4}$ & $P 2_{1} 2_{1} 2_{1}$ & $(1,0,0),(0,1,0),(0,0,1)$ \\
$(0, a, b, 0,0, c)$ & $C_{2 v}^{7}$ & $P m n 2_{1}$ & $(0,0,1),(0,1,0),(\overline{1}, 0,0)$ \\
$(a, b, 0,0, c, d)$ & $C_{i}^{1}$ & $P \overline{1}$ & $(1,0,0),(0,1,0),(0,0,1)$ \\
$(0, a, b, c, 0, d)$ & $C_{2}^{2}$ & $P 2_{1}$ & $(0,0,1),(1,0,0),(0,1,0)$ \\
$(0, a, b, 0, c, d)$ & $C_{s}^{1}$ & $P m$ & $(0,1,0),(0,0,1),(1,0,0)$ \\
$(0, a, b, c, d, 0)$ & $C_{s}^{2}$ & $P c$ & $(0, \overline{1}, 0),(1,0,0),(0,1,1)$ \\
$(a, b, c, d, e, f)$ & $C_{1}^{1}$ & $P 1$ & $(1,0,0),(0,1,0),(0,0,1)$ \\
\hline
\end{tabular}

4.

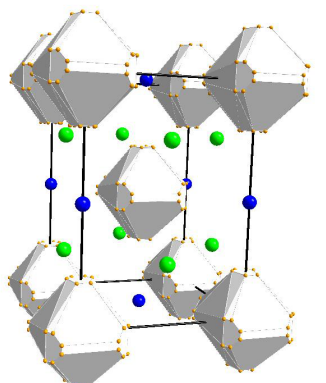

(a) $11-8, \Gamma_{5}^{-},(a, a, 0), \operatorname{Imm} 2$

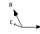

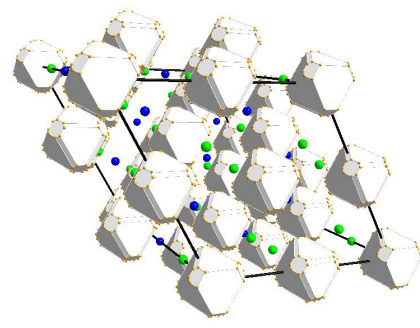

(d) $10-10, \quad \mathrm{X}_{5}^{-}, \quad(a, a, a, a, a, a)$, $R 3 m$

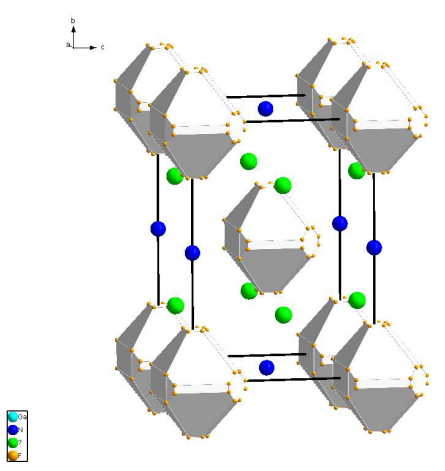

(b) $11-10, \Gamma_{4}^{-},(a, a, 0), \operatorname{Imm} 2$

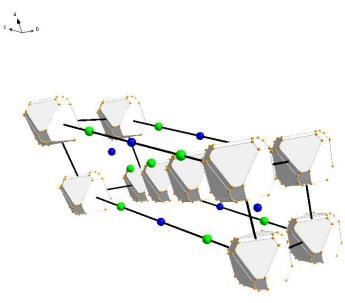

(e) $11-10, \Gamma_{4}^{-},(a, a, a), R 3 m$

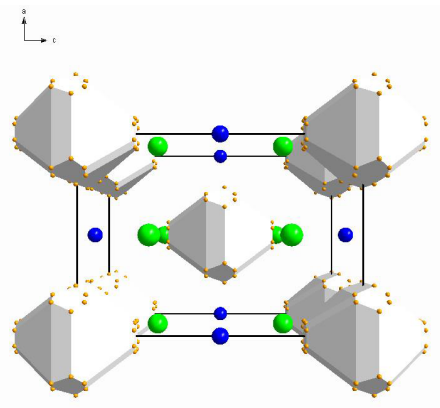

(c) $11-10, \Gamma_{4}^{-},(a, 0,0), 14 m m$

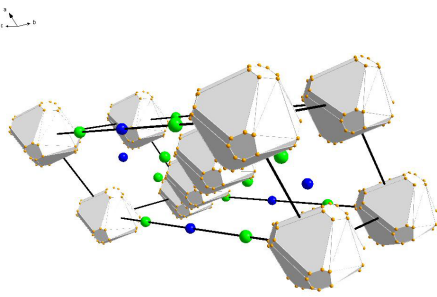

(f) $11-8, \Gamma_{5}^{-},(a, a, a), R 32$

Fig. 2. Polyhedral representation of ordered phases in elpasolite $A_{2} B B^{\prime} X_{6}$ structure 


\section{Conclusion}

Group theoretical methods derived dissymmetric phases which appear in result of $X$ anion ordering of elpasolite structure $A_{2} B B^{\prime} X_{6}$. The maximum achievable disordering of $X$ anion in $192 l$ position of cubic face-centered cell with space group $F m \overline{3} m$ were considered in the parent phase. Among all critical IR only IR of center, point $K_{11}-\Gamma$, and boundary, point $K_{10}-\mathrm{X}$, of Brillouin zone of space group $F m \overline{3} m$ were investigated. 108 dissymmetric phases were modeled during this work. Ordering of $X$ anion depend on number of components of OP which describes PT. Increasing of dimension of OP leads to decrease of influence of each component of OP on ordering process.

The research is conducted within the framework of the state task of Ministry of Education and Science of the Russian Federation to Siberian Federal University on R\&D performance in 2015 (Task 3.2534.2015/K).

\section{References}

[1] K.S.Aleksandrov, B.V.Beznosikov, Perovskite-like crystals, Novosibirsk, Nauka, 1997 (in Russian).

[2] K.S.Aleksandrov, B.V. Beznosikov, Perovskites: present and future. Variety of parent phases, phase transitions, possibilities of the synthesis of new compounds, Novosibirsk, SB RAS), 2004 (in Russian).

[3] S.V.Mel'nikova, S.V.Misyul', A.F.Bovina, M.L.Afanasev, Optical and X-ray diffraction investigations of the symmetry of distorted phases of the $\left(\mathrm{NH}_{4}\right)_{2} \mathrm{KGaF}_{6}$ crystal, Physics of the Solid State, 44(2002), no. 10, 1967-1971.

[4] I.N.Flerov, M.V.Gorev, V.D.Fokina, A.F.Bovina, M.S.Molokeev, Yu.V.Boiko, V.N.Voronov, A.G.Kocharova, Structural phase transition in elpasolite-like $\left(\mathrm{NH}_{4}\right)_{2} \mathrm{KWO}_{3} \mathrm{~F}_{3}$, Physics of the Solid State, 48(2006), no. 1, 106-112.

[5] M.V.Gorev, I.N.Flerov, A.Tressaud, D.Denu, A.I.Zaitsev, V.D.Fokina, A study of the phase diagrams of $\left(\mathrm{NH}_{4}\right)_{3} \mathrm{Ga}_{1-x} \mathrm{Sc}_{x} \mathrm{~F}_{6}$ ammonium cryolites, Physics of the Solid State, 44(2002), no. $10,1954-1960$.

[6] S.V.Mel'nikova, S.V.Misyul', A.F.Bovina, M.L.Afanas'ev, Optical and x-ray studies of the structural phase transition in cryolite $\left(\mathrm{NH}_{4}\right)_{3} \mathrm{GaF}_{6}$, Physics of the Solid State, 43(2001), no. $8,1594-1597$.

[7] T.Krahl, M.Ahrens, G.Scholz, D.Heidemann, E.Kemnitz, Structure of $\left(\mathrm{NH}_{4}\right)_{3} \mathrm{GaF}_{6}$ Investigated by Multinuclear Magic-Angle Spinning NMR Spectroscopy in Comparison with Rietveld Refinement, Inorganik Chemistry, 47(2008), no. 2, 663-670.

[8] I.N.Flerov, M.V.Gorev, V.D.Fokina, A.F.Bovina, N.M.Laptash, Calorimetric and X-ray diffraction studies of the $\left(\mathrm{NH}_{4}\right)_{3} \mathrm{WO}_{3} \mathrm{~F}_{3}$ and $\left(\mathrm{NH}_{4}\right)_{3} \mathrm{TiOF}_{5}$ perovskite-like oxyfluorides, Physics of the Solid State, 46(2004), no. 5, 915-921.

[9] M.P.Ivliev, S.V.Misyul, M.S.Molokeev, V.P.Sakhnenko, «Pseudo-proper» Ferroelectric Phase Transitions in Oxyfluoride $\mathrm{K}_{3} \mathrm{WO}_{3} \mathrm{~F}_{3}$, Phase Transitions, 87(2014), no. 6, 592-602. 
[10] K.S.Aleksandrov, S.V.Misjul, Phase Transitions Associated with Rotational Distortions in the Crystal Structure of Perovskite, Kristallografiya, 26(1981), no. 5, 1074-1085 (in Russian).

[11] S.V.Misyul, Symmetry analysis of lattice vibrations and distorted phases of the $A_{2} B B^{\prime} X_{6}$ elpasolite structure, Crystallography, 29(1984), 941-944.

[12] Y.T.Stokes, D.M.Hatch, Isotropy subgroups of the 230 crystallographic space groups, London, Wold Scientific Publishing, 1988.

[13] K.S.Aleksandrov, S.V.Misyul, E.E.Baturinets, Symmetrical Analysis of Structural Phase Transitions in Crystals with the $O_{h}^{5}$ Space Group, Ferroelectrics, 354(2007), 60-68.

[14] Yu.M.Gufan, V.P.Dmitriev, S.B.Roshal, V.I.Shezhkov, Landau phases in close-packed structures, Rostov-on-Don, Publishing house of Rostov University, 1990.

[15] O.V.Kovalev, Representation of Crystallographic Space Groups,CRC Press, 1993.

[16] S.C.Miller, W.F.Love, Tables of Irreducible Representations of The Space Groups and CoRepresentations of Magnetic Space Groups, Colorado, Pruett Press Boulder, 1967.

[17] V.P.Sakhnenko, V.M.Talanov, G.M.Chechin, Group-Theoretic Analysis of the Complete Order Parameters Condensate that Occurs at Structural Phase Transitions, Fizika Metallov i Metallovedeniya, 62(1986), no. 5, 847-856 (in Russian).

[18] H.T.Stokes, D.M.Hatch, ISOTROPY, http://stokes.byu.edu/iso/isotropy.html, 2007.

[19] K.Brandenburg, http://www.crystalimpact.com/diamond/10years.htm.

[20] International Tables for Crystallograhy. Volume: Space-group Symmetry, Edited by Theo Hahn, Kluwer Academic Publishers, 2002.

\title{
Фазы анионного упорядочения в структуре эльпасолита (упорядоченного перовскита)
}

\author{
Роман Г. Севрюков \\ Иван Н. Сафонов \\ Максим С. Молокеев \\ Сергей В. Мисюль
}

\footnotetext{
Теоретико-групповыми методами получено 108 диссимметричных фаз, возникающих в результате упорядочения анионов $X$ в структуре эльпасолита $A_{2} B B^{\prime} X_{6}$ (упорядоченного перовскита). Из критических параметров порядка рассмотренъ только такие, которые преобразуются по неприводимым представлениям точек Г и Х зоны Бриллюэна пространственной группы $\mathrm{O}_{h}^{5}-\mathrm{Fm} \overline{3} m$. Для диссимметричных фаз, переходы в которые могут сопровождатвся возникновением сегнетоэлектричества, приведены полиэдрические изображения структур. Все результаты собраны в удобных для использования таблицах.

Ключевые слова: эльпасолит, структурные данные, полный конденсат параметров порядка, неприводимые представления, критические искажения, фазовый переход, диссимметричные фа$3 b$.
} 